\title{
Further support for the alignment of cattle along magnetic field lines: reply to Hert et al.
}

\author{
S. Begall $\cdot$ H. Burda $\cdot$ J. Červený $\cdot$ O. Gerter $\cdot$ \\ J. Neef-Weisse $\cdot$ P. Němec
}

Received: 1 May 2011/Revised: 9 August 2011/Accepted: 11 August 2011/Published online: 22 October 2011

(C) The Author(s) 2011. This article is published with open access at Springerlink.com

\begin{abstract}
Hert et al. (J Comp Physiol A, 2011) challenged one part of the study by Begall et al. (PNAS 105:13451-13455, 2008) claiming that they could not replicate the finding of preferential magnetic alignment of cattle recorded in aerial images of Google Earth. However, Hert and co-authors used a different statistical approach and applied the statistics on a sample partly unsuitable to examine magnetic alignment. About $50 \%$ of their data represent noise (resolution of the images is too poor to enable unambiguous measurement of the direction of body axes, pastures are on slopes, near settlements or high voltage power-lines, etc.). Moreover, the authors have selected for their analysis only $\sim 40 \%$ of cattle that were present on the pastures analyzed. Here, we reanalyze all usable data and show that cattle significantly align their body axes in North-South direction on pastures analyzed by Hert and co-authors. This finding thus supports our previous study. In addition, we show by using aerial
\end{abstract}

Electronic supplementary material The online version of this article (doi:10.1007/s00359-011-0674-1) contains supplementary material, which is available to authorized users.

S. Begall $(\bowtie) \cdot$ H. Burda $\cdot$ O. Gerter · J. Neef-Weisse Department of General Zoology, Faculty of Biology, University of Duisburg-Essen, 45117 Essen, Germany

e-mail: sabine.begall@uni-due.de

H. Burda · J. Červený

Department of Forest Protection and Wildlife Management,

Faculty of Forestry and Wood Sciences,

Czech University of Life Sciences,

16521 Praha 6, Czech Republic

P. Němec

Department of Zoology, Faculty of Science,

Charles University in Prague, 12844 Praha 2,

Czech Republic
Google Earth images with good resolution, that the magnetic alignment is more pronounced in resting than in standing cattle.

Keywords Cattle Magnetic alignment .

Magnetoreception - Resting behavior

\section{Preamble}

In their article "No alignment of cattle along geomagnetic field lines found" published recently in J Comp Physiol A, Hert et al. (2011) challenge our study that revealed a tendency of the cattle to align their bodies along the NorthSouth axis of the magnetic field (Begall et al. 2008). The authors claim that they could not replicate this finding. However, Hert et al. used a different statistical method and applied it on a different sample. Consequently, it cannot be decided whether the disagreement between these studies is due to inadequate sampling or due to inadequate statistical evaluation in either of the two studies. We therefore checked all coordinates provided by Hert et al. (2011) in the supporting material and reanalyzed their data. Our assessment revealed that on one hand, approximately half of all pastures are not suitable for the analysis and, on the other hand, Hert et al. (2011) did not take all available cattle into account. The evaluation of usable data further supports the hypothesis of magnetic alignment in the cattle as presented by Begall et al. (2008) and Burda et al. (2009).

\section{Sampling method and sample size}

Hert et al. (2011) claim that they collected the data in a comparable way as Begall et al. (2008): "This paper 
presents a study of the body orientation of domestic cattle on free pastures in several European states, based on the Google satellite photographs. In sum, 232 herds with 3,412 individuals were evaluated.... In accordance with the way chosen in Begall et al. (2008), only animals on pastures located in horizontal areas sufficiently apart from communications and other disturbing arrangements were included in the data. No data used for evaluation overlap...". However, on checking the coordinates provided by Hert et al. in the supporting material, it is proved that their claim must be rectified.

Coordinates are given for 214 (not for 232) herds, 11 of them are duplicates (i.e. they are given twice), 25 herds are under or in close vicinity ( $<150 \mathrm{~m}$, cf. Burda et al. 2009) to power lines, at least nine pastures are on slopes of more than $10 \%$ elevation (which equals approximately $6^{\circ}$ ), at least 16 herds are on the feeder/water or on a track (noticeable by a beaten path, cattle walking in a relatively narrow row, and heading to or from a settlement or to a feeder). On 20 localities there are no herds at all, or hay bales or sheep were misinterpreted as cattle, 11 herds are on backyards or close to settlements or communications $(<25 \mathrm{~m}$, i.e. about 10 "cow lengths"), 80 coordinates (i.e., 80 herds) refer to Google Earth satellite photographs that have a resolution too poor (with a pixel length of $>50 \mathrm{~cm}$ ) to enable unambiguous determination of the body axes (notably, most of them belong to the second part of the coordinate list). In many cases, the reasons disqualifying herds from evaluation combine so that altogether 110 out of 214 given locations are useless and only 104 (i.e., less than 50\%) are usable for evaluation, of these 80 are fully and 24 only partly useful (cf. Table S1 in the supporting material to the present paper). Obviously, the authors also measured the cattle whose body orientation cannot be unambiguously recognized or even other objects representing noise.

These 104 herds usable for evaluation contain 3,830 individuals altogether. In addition, we counted 2,481 individuals in 34 more herds at the coordinates containing photographs, which were not suitable for evaluation but where the individual cattle could still be recognized. Extrapolating the average herd size of 40 animals $(6,311$ cattle on 158 pastures) on 214 herds, allegedly evaluated by Hert et al. (2011), we would expect that they measured about 8,560 individuals. Yet they have evaluated only 3,412 individuals. This discrepancy raises the question: according to which criteria Hert et al. (2011) have chosen only $40 \%$ of the available individuals and ignored $60 \%$ of them?

\section{Reanalysis and reinterpretation of the original data}

Hert et al. (2011) analyzed the distribution of the body axes in a sample of 3,412 cows and of mean values of altogether
220 herds divided into two groups. They deduce that there is no North-South alignment apparent in axial data. Besides the fact that only 104 out of those 220 herds were (partly) suitable for measurement, the authors ignore two important details. First, the $Z$ test they performed has rejected uniform distribution, i.e. random orientation of cows' body axes, at least for the subset of data called Group II. Second, judged from the figures $1 b$ and $2 b$ in their paper, cow orientation is biased towards North-South rather than East-West. This discrepancy between the data and the authors' interpretation urged us to recalculate their data using Rayleigh's uniformity test and the $V$-test (Batschelet 1981; Mardia and Jupp 2000). We copy-pasted their measurements provided in the supplementary material to Oriana and ran the tests. Strikingly, the Rayleigh's test revealed non-uniform distribution of body-axes of individual cows (total set) and a significant bias towards approximately North-South (Fig. 1). Moreover, the 95\% confidence interval for mean axis contains the North. The same is true for Group II, where significance is even higher (Fig. 1). The subset Group I was random. The $V$-test (a variant of the Rayleigh's uniformity test testing an alternative hypothesis that the distribution is a non-uniform one with a specified mean direction) confirmed that the mean axis does not significantly deviate from North ( $p=0.995$ for the total sample and $p=1$ for Group II). The Rayleigh test of the authors' data for the herds of Group II also suggests North-South alignment (mean axial vector $\mu=7^{\circ} / 187^{\circ}, p=0.064$ ).

Vector data based on cows with recognized head positions (Fig. 4 in Hert et al. 2011) are potentially more challenging, although they are not directly comparable with the data published by Begall et al. (2008). Cattle orientation in this data subset is significantly biased to the West. As the authors admit, these data were also used for axial statistics (see above). However, the axial analysis of the total dataset revealed a northward rather than a westward bias, which implies that the westward bias of the vector data has to be due to inadequate sampling, i.e. the subsample of cows with recognized head position was not representative of the whole sample.

How such bias may come about? We suggest the following explanation: head and rear are well recognizable only in lying and standing animals but not in grazing animals. In grazing animals (with their head sunken), the vector direction can be recognized unambiguously according to the shadow-however, this can be seen in cows standing more or less perpendicular to the sun only, not in cows standing parallel to the sun. Thus selection of cows with a recognizable head can easily result in a sampling bias in which the grazing cows standing parallel to the sun will be regarded as animals, in which the vector direction cannot be recognized, and thus they will be 


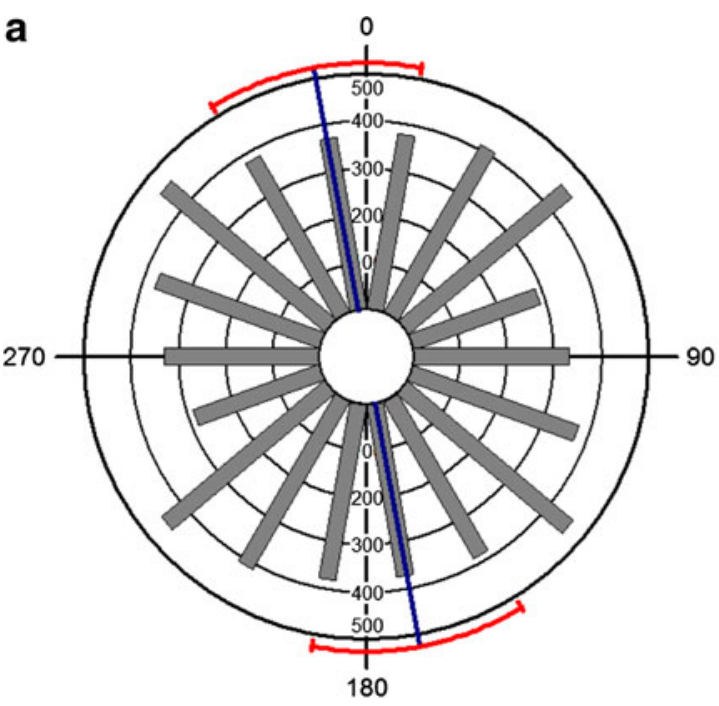

Fig. 1 Circular histograms showing the distribution of body axes of individual cows measured by Hert et al. (2011) (a) total data set (mean axis $170^{\circ} / 350^{\circ}, r=0.032, Z=3.49, p=0.03, n=3,437^{*}$ ), (b) subset Group II (mean axis $179^{\circ} / 359^{\circ}, r=0.064, Z=7.269$, $\left.p=6.9 \times 10^{-4}, n=1,801 *\right)$. Blue and red lines indicate the mean axis and $95 \%$ confidence intervals for the mean axis, respectively.

ignored. Provided more aerial images are taken from late morning till early afternoon (due to optimal light conditions) than in the early morning or late afternoon and provided the head end of the cows aligned in West-East direction will be better recognized (from their shadows), one can expect that these cows will be more represented in the sample than cows aligned North-South.

\section{Reassessment of usable data}

We measured the direction of body axis in 4,144 cattle of 122 "usable" herds from the sample of pastures, the coordinates of which were provided by Hert et al. (2011). The discrepancy to the above-stated numbers of usable data ( $n=104$ herds; 3,830 individuals) is based on the fact that some of the coordinates did not lead to a single pasture but contained several neighboring pastures. We evaluated all these separate pastures individually and included all of them in the analysis.

First, we have analyzed axial orientation of cattle following the methods described by Begall et al. (2008), except for the fact that we now measured the direction of the body axis directly on the screen using the digital ruler of the Google Earth tools. Briefly, we calculated one mean vector/ herd to obtain statistically independent data and subsequently used second-order Rayleigh test to assess clustering of the mean axis bearings. This procedure clearly rejected random distribution, the mean herd axes were significantly clustered along the North-South axis (Fig. 2a, left column,

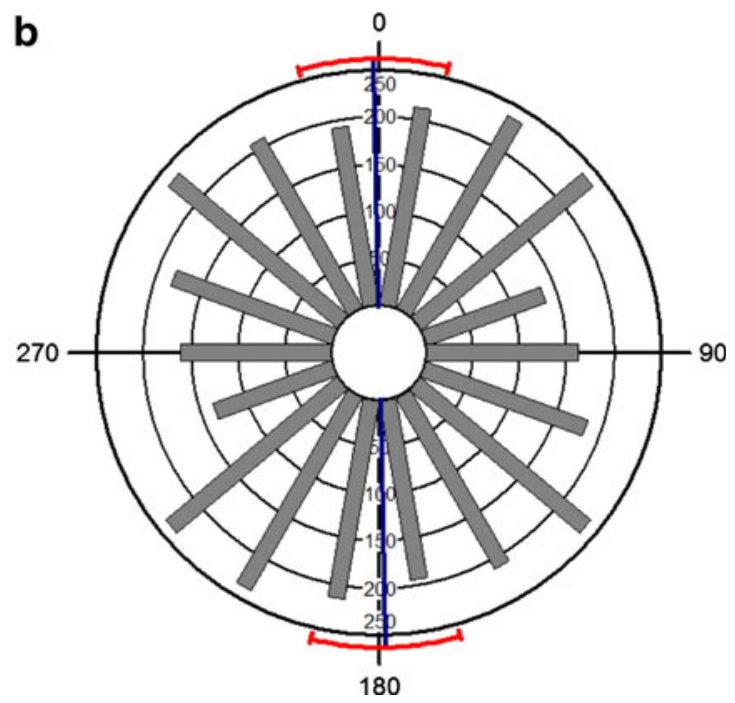

*The sample sizes indicated by Hert et al. (2001) do not match the number of axial data provided in their supplementary material. Copy and paste of the original data listed in the supplementary material resulted in a sample size difference of 25 cattle in subset Group II. Indication of the sample size for subset Group I did not differ from data set provided

and Table 1). Subsequently, we ran the same test but with exclusion of those herds, in which body orientation of individual cattle did not reach level of significance (i.e., we considered only pastures where the Rayleigh test resulted in $p<0.05$ ). To illustrate the effect of this procedure, we plotted all mean axis bearings and only significant mean axis bearings in circular diagrams for the same samples (Fig. 3). Clustering of the significant mean herd axes along the North-South axis was even more pronounced (grand mean axis $=178^{\circ} / 358^{\circ}, \quad r=0.385, Z=10.39, \quad p=3 \times 10^{-5}$, $n=70$ ). These results are not significantly different from the results provided by Begall et al. (2008) and control data provided by Burda et al. (2009). Indeed, according to the Mardia-Watson-Wheeler test the distributions of the control data from Burda et al. (2009) and the "usable" data based on Hert et al.'s sampling did not deviate significantly ( $W=4,437, p=0.109$; cf. Fig. 2a, b). Also, the mean vectors of both samples were not significantly different (Watson-Williams $F$-test: $F=1.008 ; p=0.317$ ).

Second, for the sake of comparability with the data published by Hert et al. (2011), we have also analyzed axial orientation of individual cattle (i.e. pooled data, neglecting the herds). The analysis of 4,144 individual cows resulted in even lower $p$-values and confirmed that individual cattle tend to align along approximately North-South axis (see Fig. 2a, right column, and Table 1).

Finally, we measured the orientation of the head direction in 887 cattle of 53 herds from images of good resolution enabling us to distinguish between head and rear and between lying $(n=459)$ and standing $(n=428)$ animals. 


\section{Herds}

a

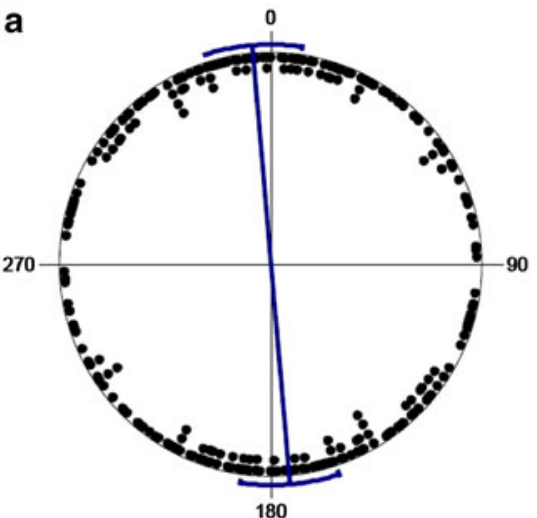

b

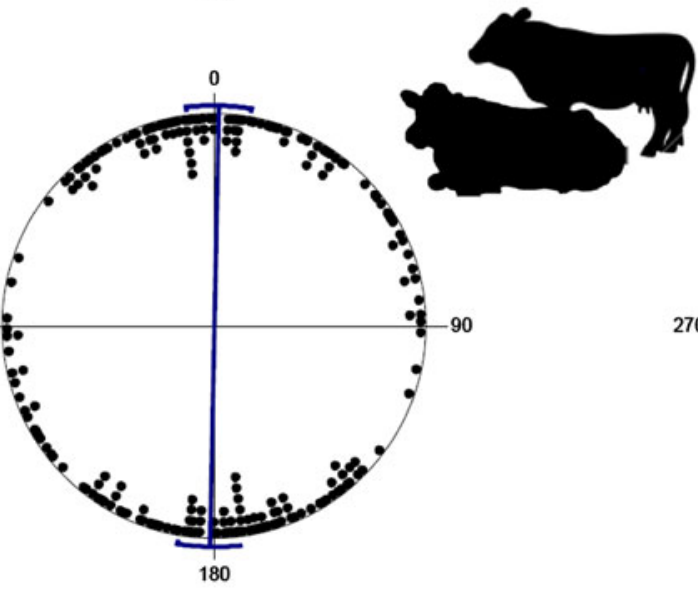

Individuals

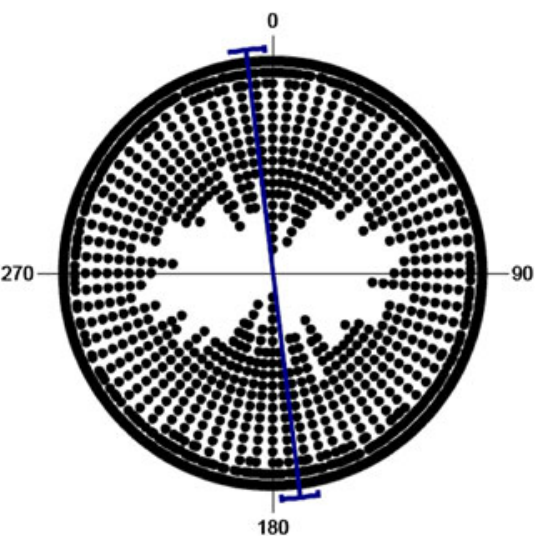

- 7 observations

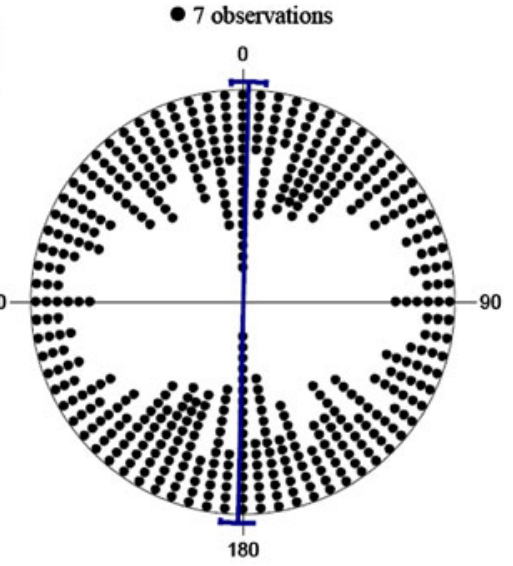

- 5 observations
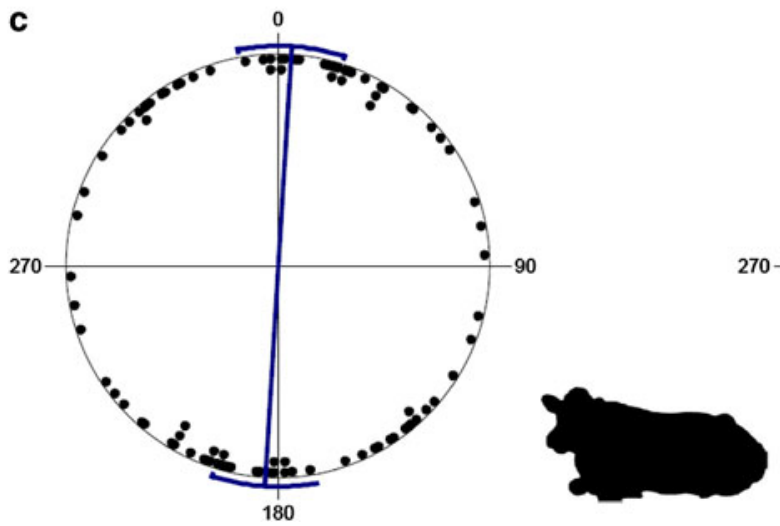

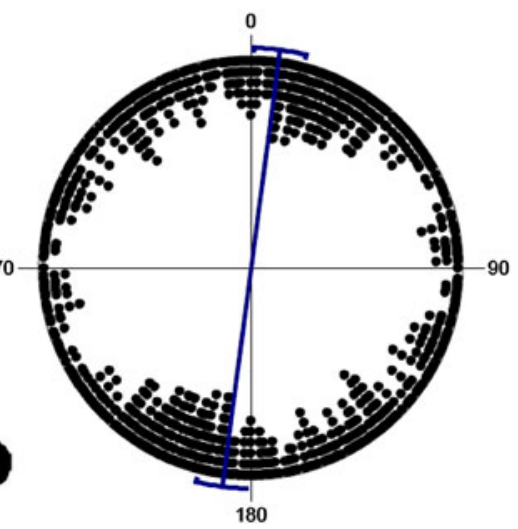

Fig. 2 Circular analysis of the body axis orientation calculated over separate herds (left column) and over individuals (right column). Mean axes and $95 \%$ confidence intervals are indicated. a Axial body orientation of 4,144 cattle (sample based on coordinates of 122 European pastures provided by Hert et al. 2011), b Axial body

Of the examined herds, 11 herds contained only lying, 5 herds only standing and 37 herds both lying and standing animals. We considered grazing animals also as standing, yet only in cases when vector direction of all the animals on the pasture could be determined (see above for orientation of 1,161 cattle from 111 European pastures used as control in the study by Burda et al. (2009). c Axial body orientation of 459 lying individuals (sample based on coordinates of 48 European pastures with reasonably high resolution provided by Hert et al. 2011). See Table 1 for supporting statistics

reasoning). Here again, for the sake of comparability with the data published by Hert et al. (2011), we have analyzed orientation of individual cattle. The Rayleigh test was used to determine whether cattle are directionally oriented. Doubling the angles and double-doubling the angles 
Table 1 Reanalysis of pastures at coordinates given in Hert et al. (2011)

\begin{tabular}{lllll}
\hline & All animals & & \multicolumn{2}{l}{ Lying animals } \\
\cline { 2 - 3 } & Means of herds & Individuals & & Means of herds \\
\hline Number of observations & 122 & 4,144 & 48 & 459 \\
Mean vector $(\mu)$ & $175^{\circ} / 355^{\circ}$ & $173^{\circ} / 353^{\circ}$ & $3 \% / 183^{\circ}$ & $8^{\circ} / 188^{\circ}$ \\
Length of mean vector $(r)$ & 0.269 & 0.129 & 0.39 & 0.266 \\
Circular SD & $46^{\circ}$ & $58^{\circ}$ & $39^{\circ}$ & $47^{\circ}$ \\
Rayleigh test $(Z)$ & 8.855 & 69.384 & 7.302 & 31.417 \\
Rayleigh test $(p)$ & $1.43 \times 10^{-4}$ & $<10^{-12}$ & $5.41 \times 10^{-4}$ & $<10^{-12}$ \\
\hline
\end{tabular}

Mean vectors refer to axial data analysis and are given as $\mathrm{XX}^{\circ} / \mathrm{XX}^{\circ} \pm 180^{\circ}$
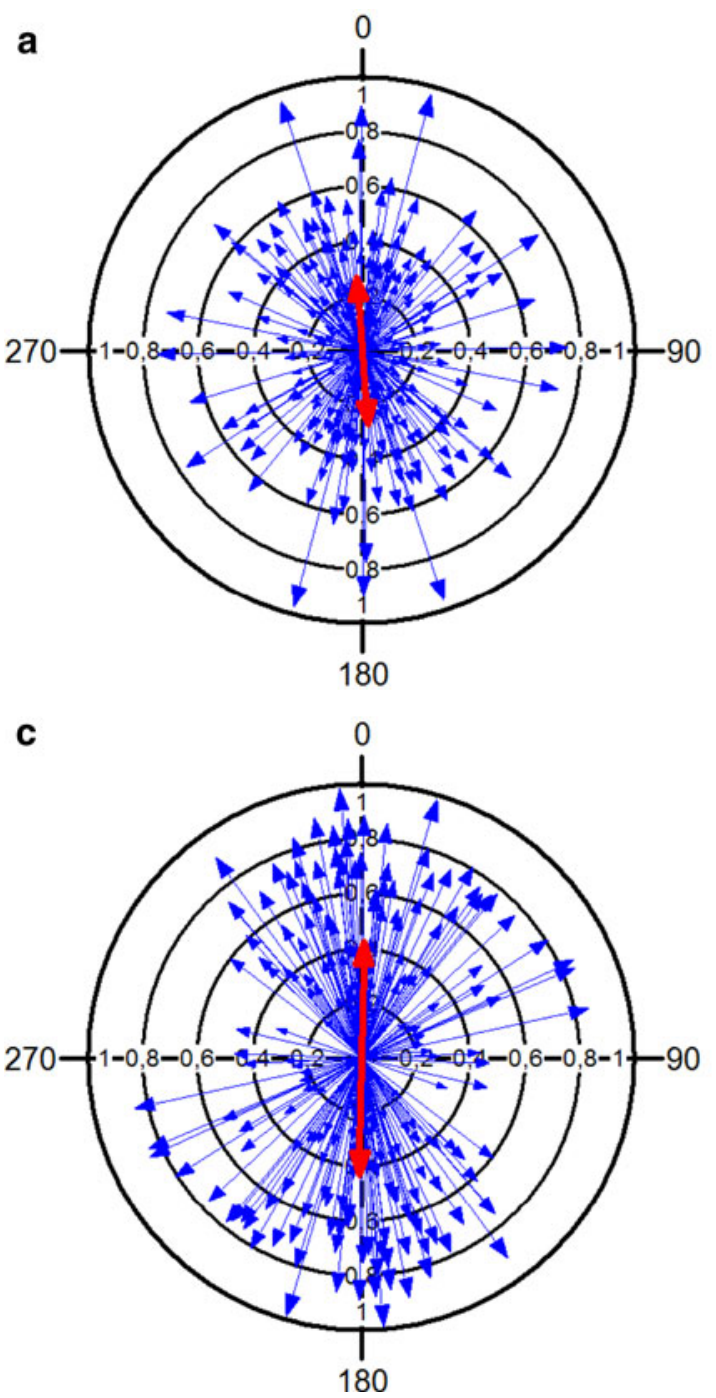

Fig. 3 Axial vector analysis of the body axis orientation calculated over separate herds. The directions of each vector pair represent the axial mean $\left(\mathrm{XX}^{\circ} / \mathrm{XX}^{\circ} \pm 180^{\circ}\right)$ of a single herd. The vector length indicates the (non-)uniformity of the herd data, i.e. the longer the vector, the more closely the individual body axes are clustered around the
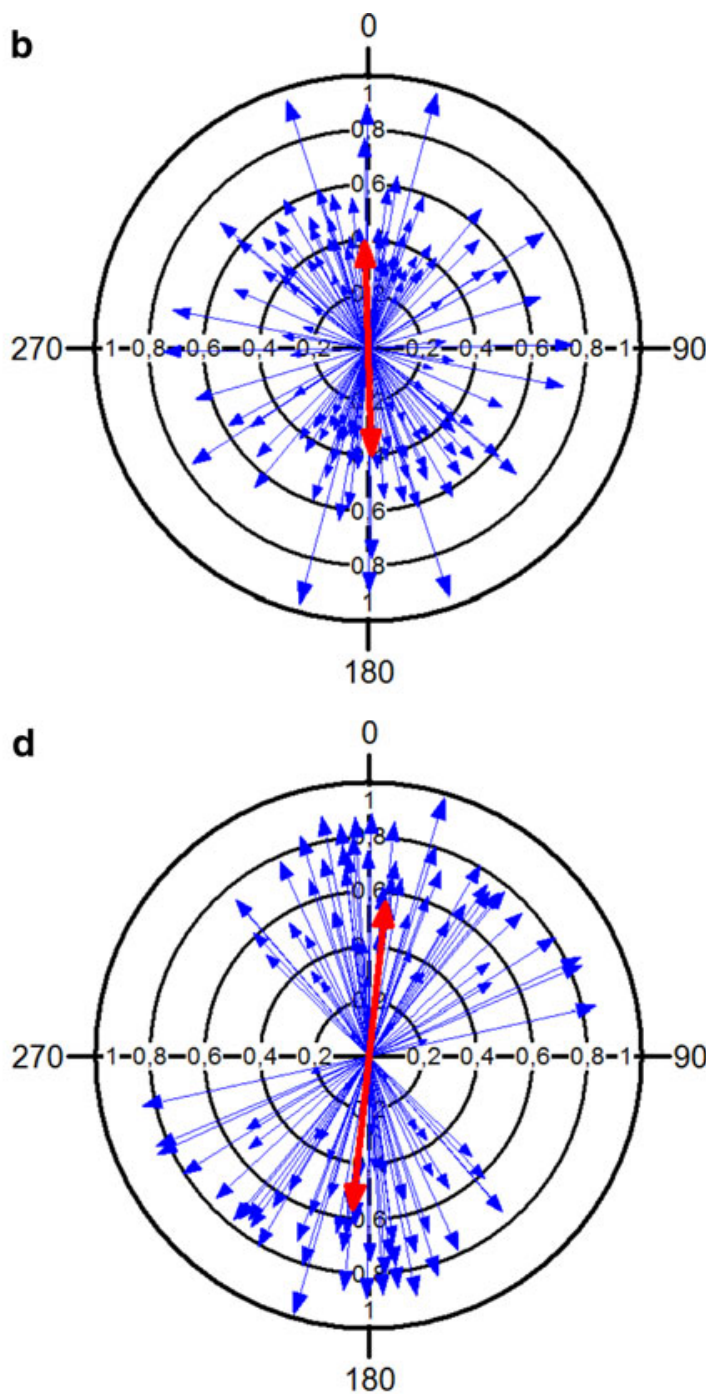

mean. Red arrows indicate grand mean axial vectors. a, b Reanalyzed data from pastures found at coordinates sampled by Hert et al. (2011); c, $\mathbf{d}$ control from Burda et al. (2009). While in a and $\mathbf{c}$ all mean values were taken into account irrespective of vector lengths, $\mathbf{b}$ and $\mathbf{d}$ contain only significant mean values $(p<0.05)$ 


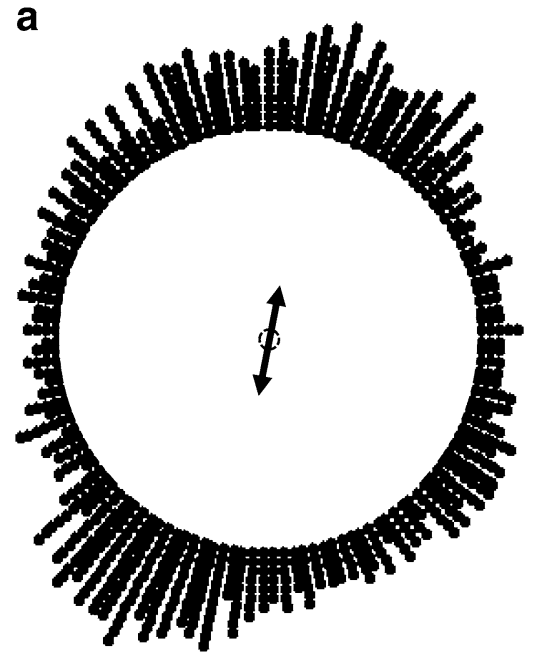

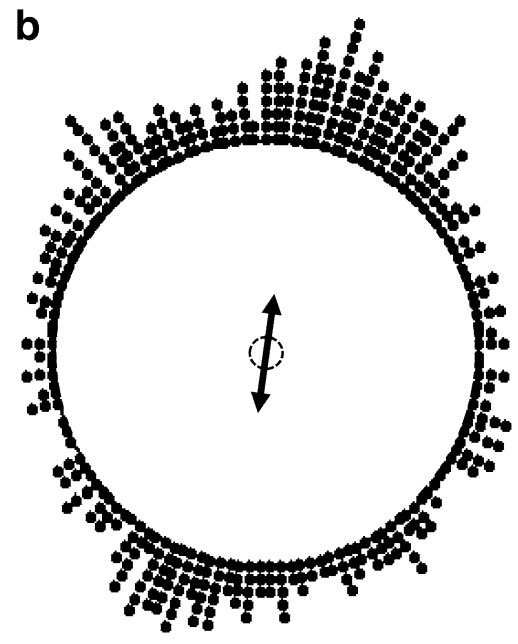

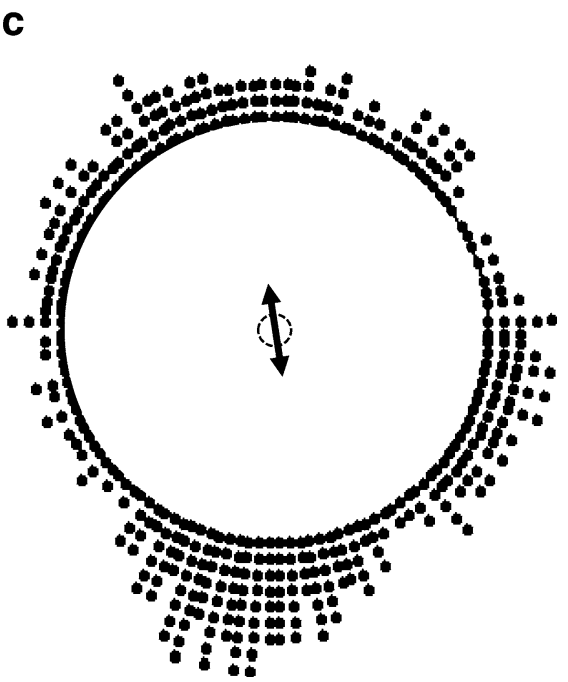

Fig. 4 Circular analysis of the angular body orientation calculated over individuals with recognized head position (sample based on coordinates of 53 European pastures with a reasonably high resolution provided by Hert et al. 2011). a herds with standing and lying cattle; b only lying animals; c only standing animals. Double-headed arrows indicate bimodal distributions ( $\left.r_{\text {bimodal }}>r_{\text {unimodal }}\right)$. The lengths of the arrows are proportional to the mean vector lengths $r$, which provides a measure of the degree of clustering in the distribution of the bearings. The inner dashed circles mark the 5\% significance border of the Rayleigh test; the arrows exceeding these circles indicate significant directional orientation. Supporting statistics: a total data set: mean vector $=11^{\circ} / 191^{\circ}, r=0.252, p<10^{-6}, n=887$, b lying animals: mean vector $=8^{\circ} / 188^{\circ}, r=0.265, p<10^{-6}, n=459$, c standing/grazing animals: mean vector $=171^{\circ} / 351^{\circ}, r=0.203$, $p<10^{-6}, n=428$ )

particularly when herds of different sizes are evaluated (e.g., a single large herd that, by chance, faces a strong wind or intentionally moves in a certain direction may significantly influence an output of statistical analysis). It should be pointed out that the size of herds analyzed by Hert et al. (2011) ranged from 10 ( 3 in one case) to almost 150 cattle. The most frequent size of the herd (11-20 cattle) was represented 47 times. The total of about 700 cows in 47 herds is, however, outweighed by just the six largest herds in the sample. As noted above, the sample included herds on tracks, under power lines, on slopes, etc. It is certainly interesting to mention in this context, that three out of these six largest herds were not suitable for testing of the hypothesis of magnetic alignment (images of two herds were at very poor resolution, one pasture was under high voltage power lines, and one herd was on a way to a water hole-cf. coordinates 20,100, 195 in the Table S1 in Supplementary material).Taken together, Hert et al.'s claim that "method with individual animals chosen as basic unit is certainly more immune against unintentional bias" is not tenable.

\section{Data interpretation: the "gap hypothesis"}

Hert and colleagues admit that the axial data of individual cows of Group II (Fig. 2b in Hert et al. 2011) have a non-random distribution (see detailed discussion above). 
Nevertheless, the authors are reluctant to interpret this distribution as North-South alignment and alternatively explain this result with the "gap hypothesis" (the authors refer in their explanation to "holes in East-West direction"). In other words, they claim that the cows do not align in North-South direction but avoid the West-East direction. In our opinion, as long as the authors do not provide any proximate or ultimate explanation why the cattle should avoid the West-East direction, this is an alternative description of the same phenomenon.

\section{Conclusion}

The data published by Hert et al. (2011) justify the conclusion that alignment of cattle with magnetic declination lines is not very tight and may be masked by many other factors influencing cattle-body orientation. However, their data by no means substantiate the rejection of the magnetic alignment hypothesis. Paradoxically enough, the subset of the data-axial distribution of body axes in cows of Group II-rather provide corroborative evidence for the hypothesis. Detailed reinvestigation of the pastures analyzed by Hert and coauthors revealed that (1) the whole study is undermined by serious flaws in data collection and sampling and (2) the cattle are aligned in approximately NorthSouth direction also on the localities chosen by the authors themselves.
Acknowledgments We thank Charlotte Schielke for help with data processing. We also thank two anonymous reviewers who helped to improve the manuscript. The study was supported by the Czech Science Foundation (project nr. 506/11/2121, to HB \& JC), Grant Agency of the Charles University (project nr. 116510, to PN) and the Ministry of Education, Youth and Sport of the Czech Republic (project nr. 0021620828, to PN).

Open Access This article is distributed under the terms of the Creative Commons Attribution Noncommercial License which permits any noncommercial use, distribution, and reproduction in any medium, provided the original author(s) and source are credited.

\section{References}

Batschelet E (1981) Circular statistics in biology. Academic Press, London, p 372

Begall S, Cerveny J, Neef J, Vojtech O, Burda H (2008) Magnetic alignment in grazing and resting cattle and deer. PNAS 105:13451-13455

Burda H, Begall S, Cerveny J, Neef J, Nemec P (2009) Extremely low-frequency electromagnetic fields disrupt magnetic alignment of ruminants. PNAS 106:5708-5713

Hert J, Jelinek L, Pekarek L, Pavlicek A (2011) No alignment of cattle along geomagnetic field lines found. J Comp Physiol A 197:677-682

Mardia KV, Jupp PE (2000) Statistics of directional data. Wiley, Chicester, p 429

Šárová R, Špinka M, Panamá JLA, Šimeček P (2010) Graded leadership by dominant animals in a herd of female beef cattle on pasture. Animal Behav 79:1037-1045 